Editorial

\title{
Postgraduate Training in Joint Surgery: The Role of Scientific Societies
}

\author{
Giuseppe Milano ${ }^{1,2}$ \\ ${ }^{1}$ Department of Orthopaedics, Catholic University, Rome, Italy \\ 2 Service of Shoulder Surgery, A. Gemelli University Hospital, \\ Rome, Italy
}

Joints 2017;5:195-196.

This issue of Joints includes an article by Losco et al, entitled "The Use and Effectiveness of the Cadaver Lab in Orthopaedic and Traumatology Education: An Italian Survey," ${ }^{1}$ which deserves particular attention.

Losco et al have conducted an interesting survey study investigating the use of cadaver laboratories in post-graduate orthopaedic training in Italy. All orthopaedic residents and trainees who attended a cadaver laboratory organized by the Società Italiana del Ginocchio Artroscopia Sport Cartilagine Tecnologie Ortopediche (SIGASCOT) between 2013 and 2016 were asked to complete a survey on the availability and current use of cadaver laboratories in the training of orthopaedic residents and trainees. Of all those who completed the interview, $44.3 \%$ had attended one or more cadaver laboratory sessions focusing on the lower limb, while $52.7 \%$ had attended one or more dealing with the upper limb.

The answers they gave revealed a remarkable level of interest in and enthusiasm for this type of training; in particular, the participants considered it particularly useful for improving knowledge of the surgical anatomy (55.7\%), surgical techniques (45.7\%) and surgical instruments, particularly arthroscopic ones (44.4\%). Over $60 \%$ of the participants felt that it had improved their surgical skills, enabling them to proceed more confidently and safely in the operating room and reducing their fear of encountering intraoperative complications.

Postgraduate surgical training courses, which in Italy are performed within the residency schools, and therefore under the responsibility of the directors of these schools, often fail to adhere to an adequate theoretical and practical teaching program, due to logistical deficiencies and a shortage of staff able to lead training activities. Therefore, the directors of these graduate schools today face tough challenges as they seek to address the substantial knowledge and experience gap of trainee medical specialists. Indeed, young trainees, on completing their course of surgical training, often express some concern about their level of autonomy and about whether their training has been sufficient to allow them to rise to the challenge of practicing their profession independently. For this reason, they welcome additional training initiatives, especially if they also involve practical training, such as cadaver laboratory sessions. ${ }^{2}$

In 2013, the Institute of Medicine (IOM) in the United States proposed a radical change in practical medicalsurgical training, suggesting that the focus be shifted away from a training pathway-based approach, which might involve evaluation based on the duration of training and on the number of operations performed, in favor of a results-based approach that, instead, takes into account the skill acquired by the trainee and the result obtained (or predicted) from a treatment performed (or suggested). ${ }^{3}$ This change effectively revolutionizes the methods of delivering training activities, making them oriented toward achievement of the final objective (a correct diagnosis and satisfactory treatment outcome), and the methods of verifying learning, which need to be able to estimate, qualitatively and quantitatively, not only the theoretical knowledge acquired by the trainee (as has been the case to date), but also his/her practical skills. To this end, numerous skill level assessment processes and instruments have recently been proposed in the field of orthopaedics, particularly in the setting of joint surgery. Some of these concern the modality for performance evaluation (virtual reality settings, plastic models, or cadaver laboratories), while others consist of evaluation tools (rating scales or checklists), which may be generic or concern specific diseases, anatomical districts or surgical procedures. ${ }^{4-8}$ This is a vast area of investigation and of research applied to education, and, providing it is underpinned by rigor and scientific method, it could revolutionize the process of medical training. Particularly in countries like Italy, whose current (unsuccessful) training model still needs to be overcome, now is an ideal time for scientific societies to spearhead the development of a new training approach that will allow medicine, both public and private, to be
Address for correspondence Giuseppe Milano, MD, Department of Orthopaedics, Service of Shoulder Surgery, A. Gemelli University Hospital, Largo Agostino Gemelli, 00136, Rome, Italy (e-mail: giuseppe.milano@unicatt.it).
DOI https://doi.org/

$10.1055 / \mathrm{s}-0037-1615789$.

ISSN 2282-4324.
Copyright @ 2017 Georg Thieme Verlag KG Stuttgart · New York
License terms

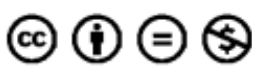


more effective and less expensive, to the satisfaction of everyone concerned, patients and healthcare professionals alike.

\section{References}

1 Losco M, Familiari F, Giron F, Papalia R. Use and effectiveness of the cadaver-lab in orthopedic and traumatology education: an Italian survey. Joints 2017;5(04):197-201

2 Martin KD, Patterson DP, Cameron KL. Arthroscopic training courses improve trainee arthroscopy skills: a simulation-based prospective trial. Arthroscopy 2016;32(11):2228-2232

3 Asch DA, Weinstein DF. Innovation in medical education. N Engl J Med 2014;371(09):794-795

4 Alvand A, Logishetty K, Middleton R, et al. Validating a global rating scale to monitor individual resident learning curves during arthroscopic knee meniscal repair. Arthroscopy 2013;29(05): 906-912

5 Hodgins JL, Veillette C, Biau D, Sonnadara R. The knee arthroscopy learning curve: quantitative assessment of surgical skills. Arthroscopy 2014;30(05):613-621

6 Cannon WD, Nicandri GT, Reinig K, Mevis H, Wittstein J. Evaluation of skill level between trainees and community orthopaedic surgeons using a virtual reality arthroscopic knee simulator. J Bone Joint Surg Am 2014;96(07):e57

7 Middleton RM, Baldwin MJ, Akhtar K, Alvand A, Rees JL. Which global rating scale? A comparison of the ASSET, BAKSSS, and IGARS for the assessment of simulated arthroscopic skills. J Bone Joint Surg Am 2016;98(01):75-81

8 Nwachukwu B, Gaudiani M, Hammann-Scala J, Ranawat A. A Checklist intervention to assess resident diagnostic knee and shoulder arthroscopic efficiency. J Surg Educ 2017;74(01): 9-15 